\title{
Artroplastia unicompartimental no tratamento da artrose medial do joelho*
}

\section{The unicompartmental arthroplasty in knee arthrosis treatment}

\author{
Gilberto luís Camanho ${ }^{1}$, Alexandre de Christo Viegas ${ }^{2}$, \\ Luís Fellipe CAMANHO ${ }^{3}$, CARina dos Reis Camanho ${ }^{4}$, ANDrea Forgas ${ }^{5}$
}

\section{RESUMO}

Objetivo: Analisar a evolução de um grupo de pacientes submetido a artroplastia unicompartimental de joelho para tratamento das artroses e nas osteonecroses que comprometem exclusivamente o comportamento medial. Métodos: Foram avaliados 49 pacientes portadores de artrose unicompartimental de joelho com idade variando entre $47 \mathrm{e}$ 88 anos de idade, sendo 12 homens e 37 mulheres. Todos foram submetidos à artroplastia unicompartimental do tipo Miller-Galante. Após seguimento mínimo de quatro anos, os pacientes foram avaliados subjetivamente pelo exame ortopédico e objetivamente pelo sistema Hospital for Special Surgery (HSS). Resultados: Apenas uma paciente necessitou de revisão para artroplastia total; quatro apresentaram dor não incapacitante; e três, queixas de instabilidade esporádica não incapacitante. Na avaliação do HSS, 30 pacientes apresentaram resultados excelentes; 18, resultados bons; e um, resultado regular. Conclusões:

* Trabalho realizado no Instituto de Ortopedia e Traumatologia da Faculdade de Medicina da Universidade de São Paulo - IOT/FMUSP e no Instituto Ortopédico Camanho - IOC.

1. Livre-Docente, Professor do Instituto de Ortopedia e Traumatologia da Faculdade de Medicina da Universidade de São Paulo - USP - São Paulo (SP), Brasil.

2. Mestre em Ortopedia e Traumatologia pela Faculdade de Medicina da Universidade de São Paulo - USP - São Paulo (SP), Brasil.

3. Médico Ortopedista do Instituto Ortopédico Camanho - São Paulo (SP), Brasil.

4. Fisioterapeuta do Instituto Ortopédico Camanho - São Paulo (SP), Brasil.

5. Pós-graduando do Instituto de Ortopedia e Traumatologia da Faculdade de Medicina da Universidade de São Paulo - USP - São Paulo (SP), Brasil.

Endereço para correspondência: Gilberto Luís Camanho, Rua Oliveira Dias, 61 - São Paulo, SP. Tel.: 3887-9776. E-mail: gilbertocamanho@uol.com.br

Recebido em 23/7/07. Aprovado para publicação em 17/9/07.

Copyright RBO2007
A artroplastia unicompartimental é uma boa opção para casos de artrose e osteonecrose do compartimento medial do joelho.

Descritores - Osteonecrose/cirurgia; Artroplastia do joelho; Articulação do joelho/patologia; Resultado de tratamento; Estudos retrospectivos

\section{ABSTRACT}

Objective: To analyze the evolution of a group of patients submitted to unicompartmental arthroplasty of the knee to treat arthrosis and in osteonecrosis that involve only the medial compartment. Methods: 49 patients with unicompartmental knee arthrosis were evaluated. Ages ranged between 47 and 88 years, 12 of them male, and 37 female. All were submitted to the Miller-Galante type unicompartmental arthroplasty. After a minimum follow-up of four years the patients were evaluated objectively by the orthopedic exam and subjectively by the HSS system. Results: Only one patient required a revision for total arthroplasty, four patients presented non-disabling pain, and three complained of sporadic instability, though not disabling. In the HSS evaluation, 30 patients had excellent results, 18 had good results, and one had a regular result. Conclusion: Unicompartmental arthroplasty is a good option for arthrosis and osteonecrosis of the medial compartment of the knee.

Keywords - Osteonecrosis/surgery; Arthroplasty, replacement, knee; Knee joint/pathology; Treatment outcome; Retrospective studies

\section{INTRODUÇÃO}

A artrose do compartimento medial do joelho, provocando uma deformidade em varo, é uma situação clínica freqüente, de etiologia em geral degenerativa. Outras causas podem le- 
var à artrose unicompartimental medial como seqüela de traumas ou sequiela de fratura por insuficiência do côndilo femoral ou tibial medial.

Por ser uma condição comum na prática clínica, tem tratamento definido: nos casos de comprometimento exclusivo do compartimento medial e em joelhos estáveis, segundo os critérios de Ahlbäck ${ }^{(1)}$, a osteotomia valgizante da tíbia é uma indicação terapêutica quase consensual; nos casos nos quais o processo degenerativo avançou e promoveu instabilidade articular, levando a deformidades compostas, o tratamento mais indicado é artroplastia total.

As osteotomias são cirurgias que exigem imobilização prolongada, tem durabilidade média em torno de oito anos e são indicadas, em geral, para pacientes relativamente jovens ${ }^{(2)}$. As artroplastias totais são cirurgias agressivas, que demandam boa condição clínica pré-operatória e períodos longos de internação e reabilitação.

A artrose unicompartimental em varo (AUV) do joelho pode acometer pacientes com idade avançada para indicação de osteotomia e com pouca degeneração dos outros compartimentos do joelho para indicação de artroplastia total.

Esse grupo de pacientes, que representa 10 a $15 \%$ dos casos de artrose unicompartimental do joelho, de causa degenerativa, tem como opção cirúrgica a substituição protética do compartimento medial, cirurgia conhecida como artroplastia unicompartimental do joelho (UNI). Essa cirurgia permite reabilitação precoce, com agressividade cirúrgica muito menor do que a artroplastia total do joelho.

Outra etiologia frequiente de artrose do compartimento medial do joelho é a seqüela da fratura por insuficiência do côndilo femoral medial, também chamada de osteonecrose do côndilo medial. A artroplastia UNI é uma boa indicação no tratamento dos pacientes de osteonecrose do côndilo femoral medial $^{(3)}$.

O objetivo deste trabalho é analisar a evolução de um grupo de pacientes submetido a UNI para tratamento da artrose unicompartimental em varo do joelho e da osteonecrose, com período de seguimento mínimo de quatro anos.

\section{MÉTODOS}

Foram operados, no Instituto de Ortopedia e Traumatologia da Faculdade de Medicina da Universidade de São Paulo, 49 pacientes com diagnóstico de AUV. Entre esses pacientes, 29 eram portadores de: artrose do compartimento medial do joelho com artrose moderada da articulação femoropatelar que não determinava dor, sem artrose do compartimento lateral e com idade acima de 65 anos (figura 1); e 20 apresentavam lesão do compartimento medial do joelho por seqüela de fratura por insuficiência do côndilo femoral medial, conhecida como osteonecrose (ON). Para inclusão nesse grupo, não houve limite de idade (figura 2).

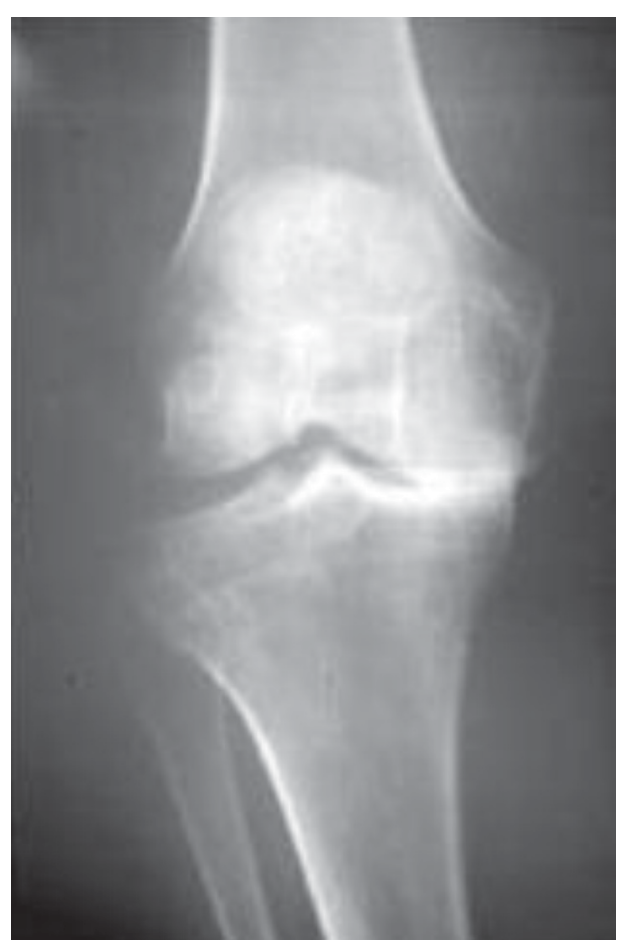

Figura 1 Artrose unicompartimental em varo do joelho

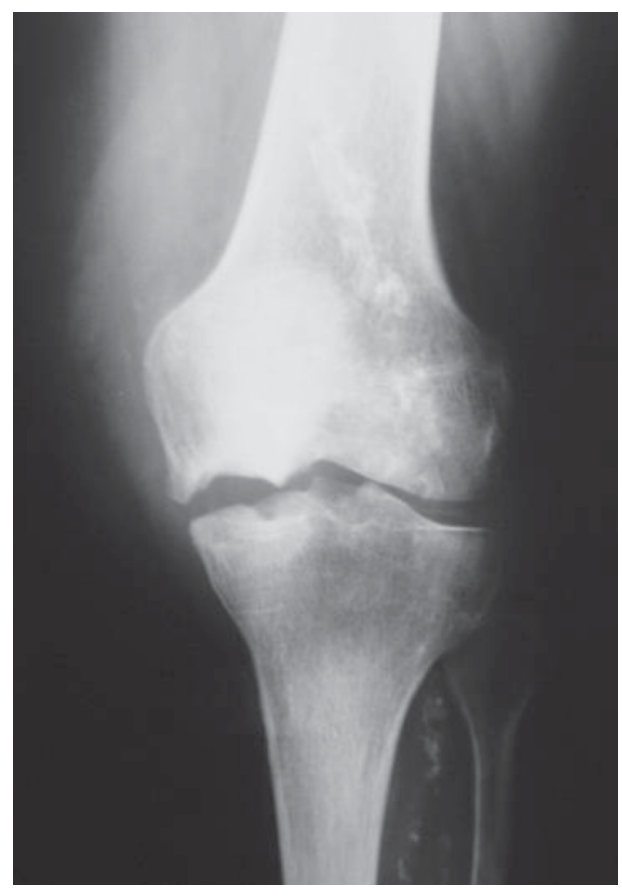

Figura 2

Osteoartrose unicompartimental do joelho, por seqüela de osteonecrose do côndilo femoral medial 
A obesidade foi considerada como contra-indicação por limitar a técnica cirúrgica.

A média de idade foi de 70 anos de idade (47-88 anos); 12 pacientes eram homens e 37, mulheres. Dos 12 pacientes homens, cinco eram portadores de ON e sete, de AUV. Das 37 mulheres, 15 eram portadores de ON e 22, de AUV.

Todos os pacientes foram operados pelo mesmo cirurgião, que utilizou prótese unicompartimental tipo Miller-Galante ${ }^{(4)}$ em todos os casos (figuras 3 e 4), e foram submetidos a tratamento anticoagulante pós-operatório preventivo. Foi utilizada enoxaparina sódica de 20 a 40mg por dia em um período de 15 dias.

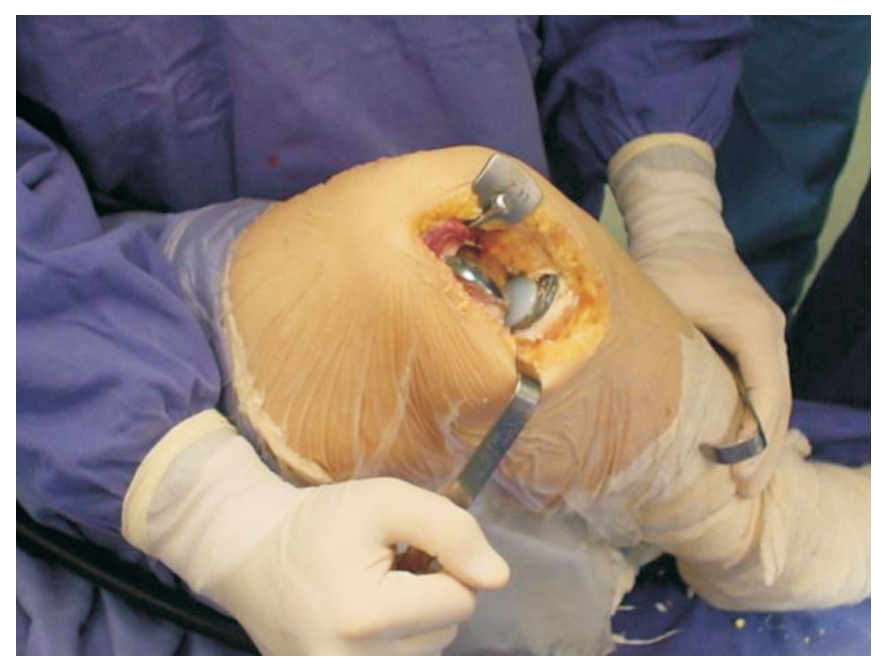

Figura 3 - Artroplastia unicompartimental com prótese MillerGalante

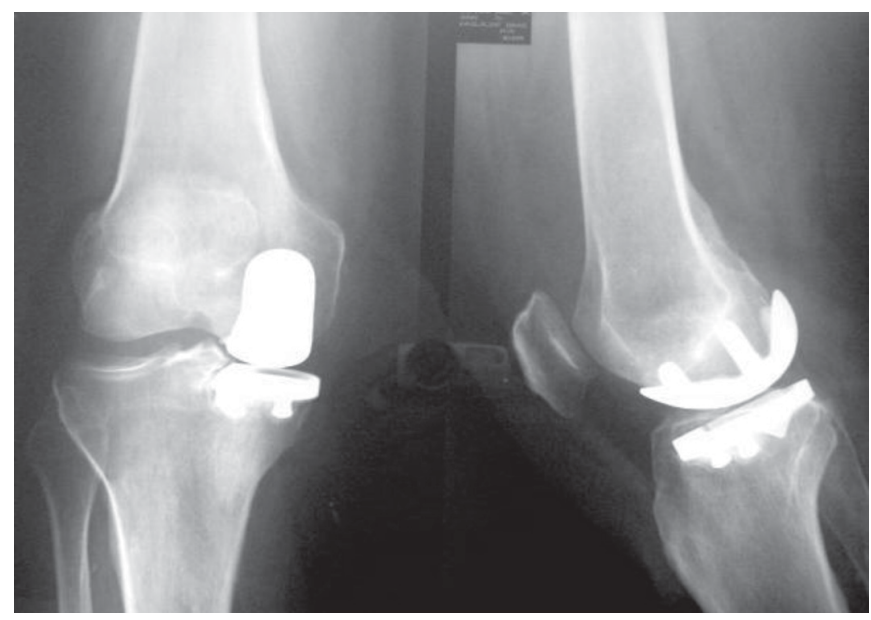

Figura 4 - Artroplastia unicompartimental feita com prótese Miller-Galante. Tempo de evolução: 74 meses.
A reabilitação foi realizada sempre no mesmo serviço de fisioterapia e constou de:

- apoio e marcha assistida por fisioterapeuta 24 horas após a cirurgia;

- alta hospitalar com autorização de marcha com apoio auxiliar (andador ou bengala) no terceiro ou quarto dia de internação;

- programa de reabilitação visando evitar a perda da extensão completa do joelho, ou seja, atitude em flexo de joelho; e ganho da amplitude de movimento da flexão do joelho;

- programa para desenvolvimento da musculatura do membro inferior e proprioceptivo.

Os pacientes estão sendo acompanhados com avaliações clínicas radiológicas semestrais pelo cirurgião ou ortopedistas de sua equipe e um fisioterapeuta, segundo o sistema Hospital for Special Surgery (HSS), como descrito por Alicea ${ }^{(5)}$.

No momento definido para confecção do presente trabalho, o menor seguimento era de 51 meses e o maior, de 123 meses.

Dois pacientes foram a óbito, eventos sem nenhuma relação com o procedimento cirúrgico, com 68 e 72 meses de seguimento pós-operatório.

\section{RESULTADOS}

Os resultados foram considerados de forma subjetiva, segundo a analise dos especialistas, e de forma objetiva, segundo o sistema $\operatorname{HSS}^{(5)}$.

\section{- Avaliação subjetiva:}

Dos pacientes, quatro apresentam queixa de dor não incapacitante, três relatam queixas esporádicas, não incapacitantes, de instabilidade ocasional, e uma apresentou queixa de dor e instabilidade e foi necessário fazer uma artroplastia total do joelho aos 48 meses de evolução (figura 5).

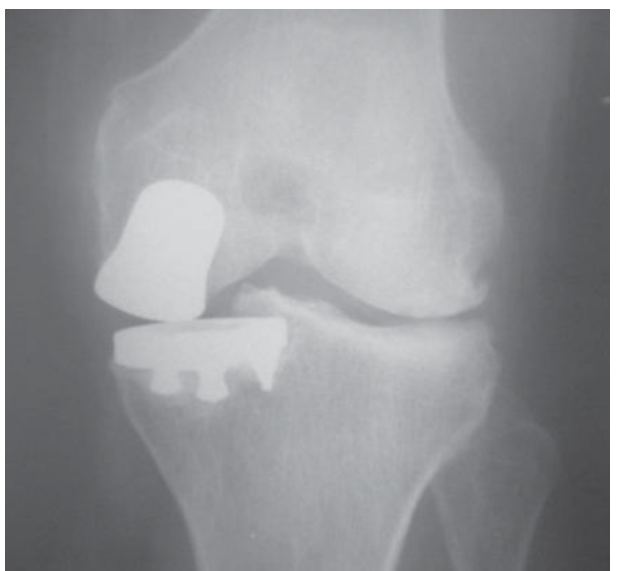

Figura 5

Radiografia de frente de artroplastia unicompartimental medial, que evoluiu para revisão 
Não houve nenhuma complicação durante o período de acompanhamento nesse grupo de pacientes.

$\mathrm{Na}$ avaliação comparativa entre os pacientes com AUV e ON, apenas um portador de ON apresentou queixa de dor não incapacitante; todas as outras queixas ocorreram no grupo AUV.

\section{- Avaliação objetiva:}

A média dos valores do HSS obtidos foi a seguinte: com valores entre 100-85, como resultado excelente, foram encontrados 30 pacientes; com resultados bons, entre $84-70$, foram encontrados 18 pacientes; e com resultados menores do que 69, apenas um paciente.

$\mathrm{Na}$ avaliação comparativa dos pacientes com AUV, 17 tiveram resultados excelentes; 11, resultados bons; e não foram encontrados pacientes com resultado regular. E dos com ON, 13 demonstraram resultados excelentes; sete, resultados bons; e um, resultado regular. Nenhum paciente teve resultado inferior a 60 , portanto, não foram encontrados resultados ruins.

\section{DISCUSSÃO}

A introdução da UNI como alternativa de tratamento das AUV e da ON é relativamente recente e ainda provoca polêmica no meio ortopédico.

A indicação cirúrgica, especialmente nos casos degenerativos, é muito rigorosa e deve ser feita dentro dos mesmos limites da indicação da osteotomia definidos por Ahlbäck ${ }^{(1)}$.

A técnica cirúrgica é mais refinada do que a utilizada para a artroplastia total do joelho. E, por ser uma indicação pouco freqüente (10 a $15 \%$ dos portadores de AUV), é quase sempre uma cirurgia realizada por cirurgião que utiliza a técnica uma ou duas vezes ao ano. Na literatura ${ }^{(5-9)}$, são raros os relatos com casuística acima de 100 pacientes. Cirurgia de indicação restrita, com ocorrência pouco freqüente, quase sempre é polêmica.

Kozinn et $a l^{(6)}$, em 1989, ou seja, nos primórdios da introdução da artroplastia unicompartimental como técnica cirúrgica, foram enfáticos nos limites de indicação para pacientes com AUV: idade superior a 60 anos, pouca atividade física, pouca dor em repouso e limitação de extensão até cinco graus.

Berend et $a l^{(10)}$ consideram o excesso de peso uma limitação importante. Relatam que, em 79 artroplastias de 69 pacientes, os resultados foram satisfatórios, porém, nos 16 casos de resultados não satisfatórios, o excesso de peso foi, de forma estatisticamente significante, responsável por uma parcela desses resultados.
A análise da nossa casuística demonstra que a média de idade para os portadores de AUV é acima dos 65 anos preconizados; a indicação para os pacientes desta série acompanhou o rigor proposto. Não tivemos pacientes que pudessem ser tidos como obesos, pois a obesidade foi considerada uma contra-indicação para a técnica cirúrgica.

Os resultados das séries iniciais de autores que utilizaram a UNI foram polêmicos e, em alguns casos, pouco encorajadores.

Veiga et al apresentam os resultados em 22 casos, relatando que, em cinco, foi necessária a conversão para artroplastia total $^{(7)}$.

Souza et $\mathrm{l}^{(8)}$ relatam os resultados precoces em 24 pacientes com seguimento precoce, em média, de 11,5 meses, com resultados insatisfatórios em $27 \%$ deles.

Nossos resultados podem ser considerados como satisfatórios, tanto nos casos de AUV como nos de ON, que já havíamos analisado $^{(3)}$.

A ocorrência de queixas esporádicas de dor e instabilidade era esperada, pois se trata de pacientes portadores de artrose, que é uma doença progressiva.

Houve necessidade de revisão em um caso. Neste, interpretamos que houve erro técnico de nossa parte no posicionamento do componente femoral (figura 5).

Nos casos em que não erramos, nem na indicação nem na técnica cirúrgica, os resultados foram aceitáveis, considerando que estamos tratando de doença evolutiva que acomete pacientes após a quinta ou sexta década de vida.

Depetris $^{(11)}$ relata sua experiência com 92 pacientes portadores de osteoartrose com graus variados e com seguimento médio de 26 meses. $\mathrm{O}$ autor relata resultados satisfatórios na maioria dos casos; apenas dois casos evoluíram para revisão com artroplastia total de joelho.

Berger $e t a l^{(9)}$ analisam a evolução de 51 pacientes, dos quais 38 viveram até 10 anos de seguimento, e relatam resultados semelhantes aos nossos. Esses autores utilizaram o mesmo sistema de UNI que empregamos, com indicação muito semelhante.

Quando avaliamos os resultados da artroplastia unicompartimental, é importante considerar o sistema que está sendo utilizado, pois não há, como na artroplastia total do joelho, uniformidade de sistemas. Outro sistema de UNI clássico é o Oxford, pouco difundido no Brasil, mas muito conhecido e utilizado na Europa ${ }^{(12)}$. Kort et al ${ }^{(12)}$, após considerar definitiva a indicação em mais idosos, relatam sua experiência em 46 pacientes com idade de 60 anos ou menos, com seguimento de dois a seis anos, apresentando bons resultados. 
Pennington et $\mathrm{l}^{(13)}$ apresentam $98 \%$ de bons resultados em pacientes com idade inferior a 60 anos utilizando o sistema Miller-Galante.

Nossa experiência em pacientes com idade inferior a 60 anos foi nos casos de ON, afecção que não tem o mesmo caráter evolutivo da artrose degenerativa.

Em nossa opinião, nos casos de artrose unicompartimental em pacientes com idade inferior a 60 anos, as osteotomias de valgização ainda são as cirurgias de eleição; entretanto, devemos estar atentos à evolução das séries que acompanham pacientes mais jovens, nas quais se utilizou a UNI como opção.

\section{CONCLUSÃO}

A análise do presente material permite concluir que a artroplastia unicompartimental do joelho é uma boa alternativa para o tratamento de artrose unicompartimental em varo e para o tratamento da osteonecrose por seqüela de fratura por insuficiência do côndilo femoral medial em joelhos estáveis, segundo os critérios de Ahlback.

\section{REFERÊNCIAS}

1. Ahlbäck S. Osteoarthrosis of the knee. A radiographic investigation. Acta Radiol Diagn (Stockh). 1968;Suppl 277:7-72.

2. Camanho GL, Olivi R, Camanho LF.Técnica de fixação para osteotomia supratuberositária cupuliforme valgizante da tibia. Rev Bras Ortop. 2001; 36(7):263-7.

3. Camanho GL, Rossetti AC, Camanho LF, Albuquerque RP. Artroplastia unicompartimental do joelho no tratamento da osteonecrose primária do côndilo femoral medial. Rev Bras Ortop. 2004;39(9):486-91.
4. Naudie D, Guerin J, Parker DA, Bourne RB, Rorabeck CH. Medial unicompartmental knee arthroplasty with the Miller-Galante prosthesis. J Bone Joint Surg Am. 2004;86-A(9):1931-5.

5. Alicea J. Scoring systems and their validation for the arthritic knee. In: Insall JN, Churchill SN, editors. Surgery of the knee. 3rd ed. New York: Livingston; 2001. p. 1507-15.

6. Kozinn SC, Scott R. Unicondylar knee arthroplasty. J Bone Joint Surg Am. 1989;71(1):145-50.

7. Veiga LT, Villardi AM, Palma IM, Vieira LAM, Leite JER, Dias MSS. Artroplastia unicompartimental do joelho: experiência após 22 casos. Rev Bras Ortop. 1997;32(5):374-6.

8. Souza GA, Queiroz AB, Pedro MAC, Navarro RD, Luzo MVM. Tratamento da osteoartrose unicompartimental medial do joelho com a utilização da prótese unicompartimental do tipo Allegretto. Resultado precoce de 26 intervenções em 24 pacientes. Rev Bras Ortop. 1998;33(2):137-44.

9. Berger RA, Meneghini RM, Jacobs JJ, Sheinkop MB, Della Valle CJ, Rosenberg AG, Galante JO. Results of unicompartmental knee arthroplasty at a minimum of ten years of follow-up. J Bone Joint Surg Am. 2005; 87(5):999-1006. Comment in: J Bone Joint Surg Am. 2006; 88(11):2533; author reply 2533-4.

10. Berend KR, Lombardi AV Jr, Mallory TH, Adams JB, Groseth KL. Early failure of minimally invasive unicompartmental knee arthroplasty is associated with obesity. Clin Orthop Relat Res. 2005;440:60-6.

11. Depetris H. [Unicompartmental knee replacement: evaluation of 103 components with 1 to 5 years follow-up]. Rev Asoc Argent Ortop Traumatol. 2004;69(2):143-8. Espanhol.

12. Kort NP, van Raay JJ, van Horn JJ. The Oxford phase III unicompartmental knee replacement in patients less than 60 years of age. Knee Surg Sports Traumatol Arthrosc. 2007;15(4):356-60.

13. Pennington DW, Swienckowski JJ, Lutes WB, Drake GN Unicompartmental knee arthroplasty in patients sixty years of age or younger. J Bone Joint Surg Am. 2003;85-A(10):1968-73. 